TRANSACTIONS OF THE

AMERICAN MATHEMATICAL SOCIETY

Volume 361, Number 11, November 2009, Pages 5695-5719

S 0002-9947(09)04705-9

Article electronically published on June 24, 2009

\title{
ANTIDIAMOND PRINCIPLES AND TOPOLOGICAL APPLICATIONS
}

\author{
TODD EISWORTH AND PETER NYIKOS
}

\begin{abstract}
We investigate some combinatorial statements that are strong enough to imply that $\diamond$ fails (hence the name antidiamonds); yet most of them are also compatible with $\mathrm{CH}$. We prove that these axioms have many consequences in set-theoretic topology, including the consistency, modulo large cardinals, of a Yes answer to a problem on linearly Lindelöf spaces posed by Arhangel'skiĭ and Buzyakova (1998).
\end{abstract}

\section{INTRODUCTION}

Researchers of set theory and allied branches of mathematics have long been familiar with the contrast between the effects of the Continuum Hypothesis $(\mathrm{CH})$ and those of MA $+\neg \mathrm{CH}$ (Martin's Axiom together with the negation of $\mathrm{CH}$ ). Also long well-known is the way the contrast is greatly magnified when $\mathrm{CH}$ is strengthened to the combinatorial axiom $\diamond$ while $\mathrm{MA}+\neg \mathrm{CH}$ is strengthened to the Proper Forcing Axiom (PFA). Until recently, however, comparatively little was known about just how much stronger $\diamond$ really is than $\mathrm{CH}$ alone. Although a great variety of mathematical structures have been defined with the help of $\diamond$, in most cases it was simply not known until recently whether or not $\mathrm{CH}$ alone is adequate to produce structures with the same basic properties.

For many years, Shelah seemed to have a near-monopoly on models of $\mathrm{CH}+\neg \diamond$, as exemplified by the $\mathbb{D}$-completeness constructions in 34 . The machinery of $\mathbb{D}$ completeness is very difficult to apply directly, while other $\mathrm{CH}+\neg \diamond$ constructions prior to 1996 were of very limited applicability. Then the first author, with the help of J. Roitman [9], introduced a generalization of $\mathbb{D}$-completeness which, with the help of some auxiliary machinery, has led to a series of very general topological statements compatible with $\mathrm{CH}$ and negated by $\diamond[6]$, [7, 8].

A bit earlier, Abraham and Todorčević [2, 3] obtained a number of PFA-like applications of a simple combinatorial axiom which they showed to be compatible with $\mathrm{CH}$. Todorčević later published new applications of a very similar but more powerful axiom [37, again compatible with $\mathrm{CH}$ but requiring the the use of large cardinals. These axioms, both designated $(*)$ in the respective articles, are far

Received by the editors July 14, 2005 and, in revised form, May 4, 2007.

2000 Mathematics Subject Classification. Primary 03E75.

Key words and phrases. Diamond, continuum hypothesis, forcing, S-space, P-ideal, antidiamond.

The first author was partially supported by a University of Northern Iowa Summer Fellowship and NSF Grant DMS-0506063.

The research of the second author was partially supported by NSF Grant DMS-9322613.

(C)2009 American Mathematical Society

Reverts to public domain 28 years from publication 5695 
simpler than the $\mathbb{D}$-completeness machinery used to establish their consistency with $\mathrm{CH}$, and much easier to apply directly than the PFA, from which they also follow [3, 37.

In this paper, we give new applications of these two axioms, and we also introduce and apply new axioms related to them. We have retained the designation of $(*)$ for the axiom of [3], but have adopted the notation $\mathcal{P}$ for the stronger axiom of [37]. They both have to do with the concept of a P-ideal:

Definition 1.1. A collection $\mathcal{I}$ of countable subsets of a set $X$ is a $P$-ideal if it is downward closed with respect to $\subset$, closed under finite union, and has the property that, if $\left\{I_{n}: n \in \omega\right\}$ is a countable subset of $\mathcal{I}$, then there exists $J \in \mathcal{I}$ such that $I_{n} \subset^{*} J$ for all $n$. [Here, $A \subset^{*} B$ means $A \backslash B$ is finite.]

Axion $\mathcal{P}$. For every P-ideal $\mathcal{I}$ on an uncountable set $X$, either

(i) there is an uncountable subset $A$ of $X$ such that every countable subset of $A$ is in $\mathcal{I}$, or

(ii) $X$ is the union of countably many sets $\left\{B_{n}: n \in \omega\right\}$ such that $B_{n} \cap I$ is finite for all $n$ and for all $I \in \mathcal{I}$.

Axiom $(*)$ of [3] is the restriction of $\mathcal{P}$ to the case where $|X| \leq \omega_{1}$. [The case where $X$ is countable trivially satisfies (ii) in ZFC.] Axiom (*) is consistent if ZFC is consistent, but all known ways of making Axiom $\mathcal{P}$ consistent make use of supercompact cardinals.

Except for Axiom $\mathcal{P}$ and a corollary $\mathcal{C C}$, all our axioms are equiconsistent with ZFC and follow from the Proper Forcing Axiom [PFA], and most are known to be compatible with $\mathrm{CH}$. The weakest axiom is designated $C C_{11}$, and (like $\mathcal{C C}$ ) it has to do with a special class of ideals "dual" to P-ideals, called countable-covering ideals. A preliminary version, 2], of [3] actually had to do with countable-covering ideals, but the authors opted in the final version for the stronger axiom, even though this made the proofs of applications slightly longer. In Section 2, we introduce the other axioms we will be applying. Sections 3,4 , and 5 focus on topological applications of Axioms $\mathcal{C C}, \mathcal{P}, P_{11}$, and $C C_{12}$ to the theory of locally compact spaces, which dovetail remarkably well with our axioms. The following theorem from Section 2 is especially noteworthy:

First trichotomy theorem [Axiom $\mathcal{C C}$. Let $X$ be a locally compact Hausdorff space. Then at least one of the following is true:

(1) $X$ is the countable union of $\omega$-bounded subspaces.

(2) X has an uncountable closed discrete subspace.

(3) $X$ has a countable subset with non-Lindelöf closure.

As a corollary of this powerful theorem, we obtain a consistent Yes answer, modulo large cardinals, to the following problem of Arhangel'skiı and Buzyakova [1]:

Problem. Is every normal locally compact linearly Lindelöf space Lindelöf?

If "locally compact" is omitted, we have one of the most basic unsolved problems in general topology, for which we do not even have any consistent answers.

In Section 6, we show how one of the strongest axioms, $P_{22}$, is compatible with $\mathrm{CH}$. 


\section{The AXIOMS AND A FEW SIMPLE APPLICATIONS}

The following concept will be useful in formulating the various axioms and clarifying implications between them.

Definition 2.1. Given an ideal $\mathcal{I}$ of subsets of a set $S$, a subset $A$ of $S$ is orthogonal to $\mathcal{I}$ if $A \cap I$ is finite for each $I \in \mathcal{I}$. The $\omega$-orthocomplement of $\mathcal{I}$ is the ideal $\{J:|J| \leq \omega, J$ is orthogonal to $\mathcal{I}\}$ and will be denoted $\mathcal{I}^{\perp}$.

When restricted to ideals whose members are countable, $\omega$-ortho-complementation is a Galois correspondence, which means that if $\mathcal{I} \subset \mathcal{J}$, then $\mathcal{J}^{\perp} \subset \mathcal{I}^{\perp}$, and that $\mathcal{I} \subset \mathcal{I}^{\perp \perp}$. This has the easy consequence that $\mathcal{I}^{\perp}=\mathcal{I}^{\perp \perp \perp}$.

Definition 2.2. The following axiom is denoted Axiom $P_{11}$ :

For every P-ideal $\mathcal{I}$ on a stationary subset $S$ of $\omega_{1}$, either

(i) there is an uncountable $A \subset S$ such that every countable subset of $A$ is in $\mathcal{I}$, or $\mathcal{I}^{\perp}$.

(ii) there is an uncountable $B \subset S$ such that every countable subset of $B$ is in

Axiom $P_{12}$ [resp. Axiom $P_{21}$ ] substitutes "stationary" for "uncountable" in (ii) [resp. (i)], while Axiom $P_{22}$ makes the same substitution in both (i) and (ii). If $\mathcal{I}$ is an ideal of countable subsets of a set $X$, then $\mathcal{I}^{\perp \perp}$ is the ideal of all countable subsets $C$ of $X$ such that for every infinite subset $D$ of $C$ there is an infinite $I \in \mathcal{I}$ such that $I \subset D$.

Like axiom $(*)$ of [3] (see the preceding section), Axiom $P_{11}$ could have been formulated using any uncountable set $X$ in place of $S$ : if $Y$ is a subset of $X$ of cardinality $\aleph_{1}$, then $\mathcal{I} \uparrow Y=\{I \cap Y: I \in \mathcal{I}\}$ is a P-ideal on $Y$, and the sets $A$ and $B$ which we obtain for $\mathcal{I}\lceil Y$ and $Y$ serve equally well for $\mathcal{I}$ and $X$.

Axiom $(*)$ of $[3]$ is a strengthening of Axiom $P_{12}$, because of the elementary fact that the union of countably many nonstationary sets is nonstationary. As shown in [3], axiom $(*)$ is compatible with $\mathrm{CH}$ even without any large cardinal assumptions, and hence so are $P_{12}$ and the weaker $P_{11}$. One of the main results of our paper is that $P_{22}$ is also compatible with $\mathrm{CH}$.

It is natural to ask whether $(*)$ or $\mathcal{P}$ in turn can be strengthened to give a stationary $A$ in (i), but the following simple example shows that this is impossible:

Example 2.3. Let $S=\omega_{1}$ and let $\mathcal{I}$ be the ideal of all countable sets of successor ordinals. Then $\mathcal{I}$ is obviously a P-ideal, and $\mathcal{I}^{\perp}$ is the ideal of all countable sets of ordinals in which all but finitely many members are limit ordinals. Obviously, no stationary subset of $\omega_{1}$ has every countable subset in $\mathcal{I}$; equally obviously, neither is $\omega_{1}$ the union of countably many sets $B_{n}$ such that $\left[B_{n}\right]^{\omega} \subset \mathcal{I}^{\perp}$ for each $n$. (As usual, $[A]^{\omega}$ stands for the set of countably infinite subsets of A.)

The foregoing example, with $\mathcal{I}$ replaced with $\mathcal{J}=\mathcal{I}^{\perp}$, shows that the axiom obtained by replacing (i) in Axiom $P_{12}$ with " $S$ is the union of countably many subsets $A_{n}$ such that every countable subset of $A_{n}$ is in $\mathcal{I}$ for all $n$ " is also inconsistent. We do not know the status of the axiom which results when the same substitution is made in Axiom $P_{11}$. In [3], it is noted that also adding the condition that the $\mathrm{P}$-ideal is $\aleph_{1}$-generated does result in a consistent axiom, but not one known to be compatible with $\mathrm{CH}$.

The remaining axioms dealt with in this paper revolve around the following concept. 
Definition 2.4. An ideal $\mathcal{J}$ of subsets of a set $X$ is countable-covering if for each $Q \in[X]^{\omega}$, the ideal $\mathcal{J} \uparrow Q$ is countably generated.

In other words, for each countable subset $Q$ of $X$, there is a countable subcollection $\left\{J_{n}^{Q}: n \in \omega\right\}$ of $\mathcal{J}$ such that every member $J$ of $\mathcal{J}$ that is a subset of $Q$ satisfies $J \subset J_{n}^{Q}$ for some $n$.

The P-ideals in all but the last section of $\underline{3}$, as well as the P-ideals in this section and Section 4 , are all $\omega$-orthocomplements of countable-covering ideals. There are no exceptions in the opposite direction:

Theorem 2.5. The $\omega$-orthocomplement of a countable-covering ideal is a P-ideal.

Proof. Let $\mathcal{J}$ be a countable-covering ideal on the set $S$ and let $\mathcal{I}=\mathcal{J}^{\perp}$. If $\left\{I_{n}: n \in \omega\right\} \subset \mathcal{I}$, let $Q=\bigcup\left\{I_{n}: n \in \omega\right\}$, and let $\left\{J_{n}: n \in \omega\right\}$ be as in Definition 2.4. Then by the Dubois-Reymond property of $\mathcal{P}(\omega) /$ fin, there is a subset $H$ of $Q$ such that $I_{n} \subset^{*} H$ for all $n \in \omega$ while $J_{n} \cap H$ is finite for all $n$. It is easy to see that $H \in \mathcal{J}^{\perp}$, as required.

A nice feature of countable-covering ideals on a set of cardinality $\omega_{1}$ is that they are $\aleph_{1}$-generated; that is, each one has a cofinal subset of cardinality $\omega_{1}$ with respect to $\subset$. Another nice feature is given by:

Theorem 2.6. If $\mathcal{J}$ is countable-covering, then $\mathcal{J}=\mathcal{J}^{\perp \perp}$.

Proof. Suppose $Q$ is a countable subset of the underlying set $S$ and $Q \notin \mathcal{J}$. Let $\left\{J_{n}: n \in \omega\right\} \subset \mathcal{J}$ be such that every member $J$ of $\mathcal{J}$ that is a subset of $Q$ satisfies $J \subseteq \subseteq^{*} J_{n}$ for some $n$. Since $Q \notin \mathcal{J}$ we can define, by induction, elements $\left\{q_{n}: n \in \omega\right\}$ such that $q_{n} \notin J_{0} \cup \cdots \cup J_{n}$ for all $n$. Then $\left\{q_{n}: n \in \omega\right\} \in \mathcal{J}^{\perp}$, so that $Q \notin \mathcal{J}^{\perp \perp}$, as desired.

These last two theorems make it easy to see that the following axioms are weaker than the corresponding $P_{m n}$ axioms with the subscripts reversed.

Definition 2.7. Axiom $C C_{11}$ is the axiom where for each countable-covering ideal $\mathcal{J}$ on a stationary subset $S$ of $\omega_{1}$, either:

(i) there is an uncountable $A \subset S$ such that $[A]^{\omega} \subset \mathcal{J}$; or

(ii) there is an uncountable $B \subset S$ such that $[B]^{\omega} \subset \mathcal{J}^{\perp}$.

Similarly, Axioms $C C_{12}, C C_{21}$ and $C C_{22}$ are defined analogously to the corresponding $P_{m n}$ axioms. If $\mathcal{J}$ is a countable-covering ideal, then Theorem 2.5 tells us that $\mathcal{I}=\mathcal{J}^{\perp}$ is a P-ideal and Theorem 2.6 tells us that $\mathcal{I}^{\perp}=\mathcal{J}$. It follows immediately that $P_{n k}$ implies $C C_{k n}$ for each choice of $k$ and $n$. Obviously, the $P_{n k}$ and $C_{k n}$ axioms also increase in strength with an increasing subscript, but at our present state of knowledge we do not know what other implications hold between them. We do not even know whether $C_{11}$ implies $P_{22}$ or $(*)$. Nevertheless, almost every application of these axioms has a "naturally weakest" axiom associated with it, in the sense that one cannot improve on the proof by going to a stronger axiom in this set, and no formally weaker axiom in the set yields a similar proof.

For example, the "naturally weakest" axiom for all but one of the consequences of (*) in [3] is $C C_{11}$. (The exception is Definition 2.4, for which the countable-covering counterpart of $(*)$ is the natural weakest.) Thus $C C_{11}$ suffices for the nonexistence of Souslin trees, the statement that every $\left(\omega_{1}, \omega_{1}^{*}\right)$-gap contains a Hausdorff gap, and a similar result about coherent sequences on $\omega_{1}$. In each case, the proof is actually a bit simpler when $C C_{11}$ is used. 
The following axioms are known not to be equivalent to any considered thus far.

Definition 2.8. Axiom $P_{31}$ [resp. Axiom $\mathrm{CC}_{13}$ ] is the following axiom.

For every P-ideal $\mathcal{I}$ [resp. for every countably covering ideal $\mathcal{J}]$ on $\omega_{1}$, either

(i) there is a closed unbounded $A \subset \omega_{1}$ such that every countable subset of $A$ is in $\mathcal{I}$ [resp. in $\mathcal{J}^{\perp}$ ], or

(ii) there is an uncountable $B \subset \omega_{1}$ such that every countable subset of $B$ is in $\mathcal{I}^{\perp}$ [resp. in $\left.\mathcal{J}\right]$.

Axiom $P_{32}$ [resp. Axiom $C_{23}$ ] is obtained from Axiom $P_{31}$ [resp. Axiom $C C_{13}$ ] by substituting "stationary" for "uncountable" in (ii).

We do not yet know whether any or all of the axioms in Definition 2.8 are compatible with $\mathrm{CH}$. This was claimed in an earlier version of [14, where $P_{32}$ is designated " $\left(*_{c}\right)$ for $\theta=\omega_{1}$," but the proof was faulty. However, this axiom does follow from the PFA [14. Also, as will be shown in a future paper, substituting a stationary set for $\omega_{1}$ in each of the axioms in Definition 2.8 gives an axiom which is compatible with $\mathrm{CH}$. In contrast, Hirschorn has a simple counterexample in 2.3 of [14] that would logically be denoted Axiom $P_{13}$, and it also serves as a counterexample that one would call $C C_{31}$. In the same article it is shown how $\left(*_{c}\right)$ for $\theta=\omega_{1}\left(=P_{32}\right)$ implies that every Aronszajn tree is special, and that it negates what is there called "the existence of a club-guessing sequence on $\omega_{1}$ " and is called $\boldsymbol{q}_{C}$ below. It is easy to modify the proofs to show that the weaker $C C_{13}$ implies these things also; we do it explicitly for $\boldsymbol{\beta}_{C}$ below.

Definition 2.9. A ladder system on $\omega_{1}$ is a family $\left\{S_{\alpha}: \alpha \in \omega_{1} \cap \operatorname{Lim}\right\}$ of sets of order type $\omega$ such that $S_{\alpha}$, called the ladder at $\alpha$, has supremum $\alpha$.

The axiom $\left[\right.$ resp. $\left.\boldsymbol{q}_{S}\right]\left[\right.$ resp. $\left.\boldsymbol{q}_{C}\right]$ states that there is a ladder system $\left\{S_{\alpha}: \alpha \in\right.$ $\left.\omega_{1} \cap \operatorname{Lim}\right\}$ on $\omega_{1}$ such that for every uncountable [resp. stationary] [resp. closed unbounded ("club")] subset $E$ of $\omega_{1}$, there exists $\alpha$ such that $S_{\alpha} \subset E$ for some $\alpha<\omega_{1}$.

It is a well-known fact that $\boldsymbol{\rho}_{C}$ is compatible with $M A+\neg C H$; in fact, $\boldsymbol{\phi}_{C}$ cannot be destroyed by ccc forcing. Much more strongly, it cannot even be destroyed by $\omega$-proper forcing [15. Now, all axioms considered in this paper (as well as in conjunction with $\mathrm{CH}$ ) except for those in Definition 2.8 and Theorem 5.1 can be shown to be consistent using $\alpha$-proper forcing for all $\alpha \leq \omega_{1}$. Thus our other applications are compatible with $\boldsymbol{p}_{C}$.

\section{Theorem 2.10.}

(a) $C C_{11} \Longrightarrow \neg$ p.

(b) $C C_{12} \Longrightarrow \neg \boldsymbol{p}_{S}$.

(c) $C C_{13} \Longrightarrow \neg \boldsymbol{p}_{C}$.

Proof. Let $\mathcal{J}$ be the ideal generated by the sets $S_{\alpha}$. This is countable-covering; in fact, $\mathcal{J}\left\lceil Q\right.$ is countable for all countable $Q \subset \omega_{1}$. Now, it is impossible for there to be a set of order type $\geq \omega^{2}$ in $\mathcal{J}$, so alternative (i) fails in $C C_{1 n}$. But alternative (ii) gives an uncountable [resp. stationary] [resp. club] subset of $\omega_{1}$ which meets each ladder $S_{\alpha}$ in a finite set, very strongly negating the respective variants of

Problem 1. Does $C C_{11}$ or $C C_{21}$ imply $\neg \boldsymbol{q}_{S}$ ?

One can ask the same questions about the corresponding $P_{n m}$ axioms here and in the next problem. 
Problem 2. Does $C C_{11}$ or $C C_{21}$ imply that every Aronszajn tree has an antichain that meets a stationary set of levels?

In [4 an Aronszajn tree is called "almost Souslin" if every antichain meets a nonstationary set of levels. Thus Problem 2 can be rephrased as "Does $C C_{11}$ or $C C_{21}$ imply there are no almost Souslin trees?" In [35] and [36] there are models of Souslin's Hypothesis in which there do exist almost Souslin trees. In contrast, it is unknown whether $\boldsymbol{\phi}$ and $\boldsymbol{\phi}_{S}$ are distinct axioms - does $\boldsymbol{\phi}_{S}$ actually imply $\boldsymbol{中}$ ?

Problem 3. Does any or all of $C C_{11}$ through $P_{22}$ imply that every Aronszajn tree is special?

\section{TRICHOTOMIES ON LOCALLY COMPACT SPACES}

In this section we give several powerful applications of Axiom $\mathcal{P}$ and the slightly weaker Axiom $\mathcal{C C}$ to the theory of locally compact spaces. The applications revolve around the ideal $\mathcal{I}$ of countable closed discrete subspaces and the ideal $\mathcal{J}$ of countable subsets with compact closures. If $X$ is a locally compact Hausdorff space, then $\mathcal{I}=\mathcal{J}^{\perp}$ because a countable subset of $X$ is in $\mathcal{J}^{\perp}$ iff it meets every open set with compact closure in a finite set iff it is closed discrete. Also, $\mathcal{J}^{\perp \perp}$ is the ideal of all countable subsets $Q$ of $X$ such that every infinite subset of $Q$ has an accumulation point in $X$ (though not necessarily in $Q$ itself). Because of the Galois correspondence between $\mathcal{I}$ and $\mathcal{J}$, we have $\mathcal{I}^{\perp \perp}=\mathcal{I}$.

Axiom $\mathcal{C C}$ is to $\mathcal{P}$ as $C C_{m n}$ is to $P_{n m}$ :

Axiom $\mathcal{P}$ [resp. Axiom $\mathcal{C} C$. For every P-ideal $\mathcal{I}$ [resp. For every countablecovering ideal $\mathcal{J}$ ] on a set $X$, either

(i) there is an uncountable subset $A$ of $X$ such that every countable subset of $A$ is in $\mathcal{I}$ [resp. in $\left.\mathcal{J}^{\perp}\right]$, or

(ii) $X$ is the union of countably many sets $\left\{B_{n}: n \in \omega\right\}$ such that every countable subset of each $B_{n}$ is in $\mathcal{I}^{\perp}$ [resp. in $\mathcal{J}$ ].

It follows from Theorem 2.5 that $\mathcal{P}$ implies $\mathcal{C C}$; the converse is an open problem.

The following lemma leads to a far-reaching trichotomy with the help of Axiom $\mathcal{C C}$. Recall that a space is called Lindelöf if every open cover has a countable subcover. Obviously, every $\sigma$-compact space is Lindelöf.

Lemma 3.1. Let $X$ be a locally compact Hausdorff space and let $\mathcal{J}$ be the ideal of all countable subsets of $X$ with compact closure. Then $\mathcal{J}$ is countable-covering if, and only if, every countable subset of $X$ has Lindelöf closure.

Proof. If the countable subset $Q$ has Lindelöf closure in $X$, let $\left\{V_{n}: n \in \omega\right\}$ be an ascending cover of $\bar{Q}$ by open subsets of $X$ with compact closures. Let $J_{n}=V_{n} \cap Q$ for all $n$. Then $J_{n} \in \mathcal{J}$ for all $n$. Now suppose that $J \in \mathcal{J}$ and $J \subset Q$. Since $\bar{J}$ is a subset of $\bar{Q}$ and is compact, $\bar{J} \subset V_{n}$ for some $n$, and so $J \subset J_{n}$.

For the converse, let $Q$ be a countable subset of $X$ and suppose that $\mathcal{J}\lceil Q$ is generated by $\left\{J_{n}: n \in \omega\right\}$. We may assume that the $J_{n}$ form an ascending family. It suffices to show that $\bar{Q} \subset \bigcup\left\{\overline{J_{n}}: n \in \omega\right\}$ since each $\overline{J_{n}}$ is compact. For each point $p$ of $\bar{Q}$ let $V$ be an open neighborhood of $p$ in the relative topology of $\bar{Q}$ such that $\bar{V}$ is compact. $Q$ is dense in $\bar{Q}$, hence $\overline{V \cap Q}=\bar{V}$. Since $V \cap Q$ has compact closure, $V \cap Q \subset J_{n}$ for some $n$, and so $p \in V \subset \overline{J_{n}}$. 
Our applications of Axiom $\mathcal{C C}$ involve the following concepts:

Definition 3.2. A space $X$ is $\omega$-bounded if every countable subset has compact closure. A space $X$ is pseudocompact if every continuous real-valued function on $X$ is bounded. A space $X$ is linearly Lindelöf if every open cover that is totally ordered by $\subset$ has a countable subcover. A space $X$ is countably metacompact if every countable open cover has a point-finite open refinement.

Here are some basic facts about these concepts; all except the last two have very easy proofs.

- Every $\omega$-bounded space is countably compact (that is, every infinite subset has an accumulation point).

- Every countably compact $T_{1}$ space is countably metacompact, and so is every $T_{1}$ space that is the countable union of closed, countably compact subspaces.

- Every countably compact space is pseudocompact; more generally, so is every space with a dense countably compact (or pseudocompact) subspace.

- Every normal, pseudocompact space is countably compact [10] (p. 206).

- Every countably metacompact, linearly Lindelöf space is Lindelöf [13].

In this paper, "regular" and "normal" are both taken to include the Hausdorff property.

Theorem 3.3 (First Trichotomy Theorem [Axiom $\mathcal{C C}]$ ). Let $X$ be a locally compact Hausdorff space. Then at least one of the following is true:

(1) $X$ is the countable union of $\omega$-bounded subspaces.

(2) $X$ has an uncountable closed discrete subspace.

(3) X has a countable subset with non-Lindelöf closure.

Proof. If (3) fails, it follows from Lemma 3.1 that the ideal $\mathcal{J}$ of countable subsets of $X$ with compact closure is countable-covering. Then Axiom $\mathcal{C C}$ kicks in to give either (a) an uncountable subset $A$ of $X$ in which every countable subset is closed discrete in $X$ or (b) a cover of $X$ by countably many sets $B_{n}$ such that $B_{n}$ meets every closed discrete subset of $X$ in a finite set. Then, in case (a), local compactness gives that $A$ is closed discrete: any compact neighborhood of a point can only meet $A$ in a finite set. Thus alternative (2) holds. In case (b), every infinite subset of $B_{n}$ has a limit point somewhere in $X$. Let $A_{n}=\bigcup\left\{\bar{Q}: Q\right.$ is a countable subset of $\left.B_{n}\right\}$.

Claim. $A_{n}$ is $\omega$-bounded. Once the Claim is proven, alternative (1) holds and we are done.

Proof of the Claim. Let $R$ be a countable subset of $A_{n}$; then $R$ is a subset of the closure of a countable subset $Q$ of $B_{n}$, and $Q$ has Lindelöf closure. Also, $\bar{Q}$ is pseudocompact, because every sequence in $Q$ has an accumulation point in $\bar{Q}$, and it is Tychonoff because it is locally compact and Hausdorff. Now use the well-known fact that every pseudocompact, Tychonoff, Lindelöf space is compact.

Every known model of $\mathcal{C C}$ has $\mathfrak{c}$ (the cardinality of the continuum) equal either to $\aleph_{1}$ or $\aleph_{2}$, so the cardinality restriction in the our next theorem may be redundant:

Theorem $3.4\left(\mathcal{C C}+\mathfrak{c}<\aleph_{\omega}\right)$. Every locally compact, normal, linearly Lindelöf space is Lindelöf.

Proof. Alternative (2) in Theorem 3.3 cannot hold in a linearly Lindelöf space: if $D$ is an uncountable closed discrete subspace of a space $X$, let $\left\{d_{\xi}: \xi<\omega_{1}\right\}$ list 
$\aleph_{1}$ points of $D$, let $E=X \backslash\left\{d_{\xi}: \xi<\omega_{1}\right\}$ and let $U_{\alpha}=E \cup\left\{d_{\xi}: \xi<\alpha\right\}$ for all $\alpha<\omega_{1}$. Then $\left\{U_{\alpha}: \alpha<\omega_{1}\right\}$ is an open cover with no countable subcover.

Alternative (3) cannot hold in a regular linearly Lindelöf space if $\mathfrak{c}<\aleph_{\omega}$. For then, the closure of any countable subset has a base of cardinality less than $\aleph_{\omega}$, and in a linearly Lindelöf space, every open cover of cardinality $<\aleph_{\omega}$ has a countable subcover [13. Clearly, every closed subspace of a linearly Lindelöf space is linearly Lindelöf, so the closure is Lindelöf.

So now we turn to alternative (1). Let $X$ be a linearly Lindelöf space which is the union of countably many $\omega$-bounded subspaces $X_{n}$. The closure $\overline{X_{n}}$ is pseudocompact since $X_{n}$ is a dense countably compact subspace. If $X$ is normal, so is each $\overline{X_{n}}$. Hence $X=\bigcup_{n} \overline{X_{n}}$ is the countable union of closed countably compact subspaces, and is thus countably metacompact. It now follows that $X$ is Lindelöf.

In the foregoing proof, the only use of normality was in showing that the subspaces $\overline{X_{n}}$ are countably compact. Two general properties that lead to the same conclusion are countable tightness and the wD property:

Definition 3.5. A space $X$ is countably tight if

$$
\bar{A}=\bigcup\{\bar{Q}: Q \subset A, \quad Q \text { is countable }\}
$$

for all subsets $A$ of $X$. A space $X$ satisfies Property $w D$ if for each infinite closed discrete subset $A$ there is an infinite $D \subset A$ such that $D$ has an expansion to a discrete family $\left\{U_{d}: d \in D\right\}$ of open sets. [To say that $\left\{U_{d}: d \in D\right\}$ is an expansion of $D$ is to mean that $U_{d} \cap D=\{d\}$ for all $d \in D$.]

Clearly, every first countable or sequential space is countably tight; so is every hereditarily separable space. Spaces satisfying wD include normal spaces and realcompact spaces.

In particular, it is well known that pseudocompact, realcompact spaces are compact. Thus we can substitute "realcompact" for "normal" in Theorem 3.4 thus giving a consistent answer, modulo large cardinals, to the following problem, Question $16=$ Problem 482 of [28]:

Problem. Is every realcompact, locally compact, linearly Lindelöf space Lindelöf?

Theorem 3.6 (Axiom $\mathcal{C C}$ ). Let $X$ be a locally compact Hausdorff space. If $X$ is countably tight or has property $w D$, then at least one of the following is true:

$\left(1^{+}\right) X$ is the union of countably many closed $\omega$-bounded, hence countably compact subspaces.

(2) $X$ contains an uncountable closed discrete subspace.

(3) $X$ has a countable subset with non-Lindelöf closure.

Proof. It is easy to see that every $\omega$-bounded subset of a countably tight Hausdorff space is closed. So $\left(1^{+}\right)$follows from (1) of Theorem 3.3 in this case. If $Y$ is a countably compact subspace of $X$, then $\bar{Y}$ is pseudocompact. It is well known (cf. [10]) and easy to show that every discrete family of open sets in a pseudocompact Tychonoff space is finite; hence every pseudocompact Tychonoff space satisfying wD is countably compact. If (3) fails, then every countable subset of $\bar{Y}$ has compact closure; hence $\left(1^{+}\right)$follows from (1) in this case too, and we can apply Theorem 3.3 . 
Some properties of $X$ besides being just locally compact and Hausdorff are needed in Theorem 3.6. K. Kunen has constructed a ZFC example of locally compact, Hausdorff, linearly Lindelöf, non-Lindelöf space [19. From the proof of Theorem 3.4 and the ensuing discussion, it is clear that $\left(1^{+}\right),(2)$, and (3) all fail in Kunen's space. It is a countable union of dense $\omega$-bounded subspaces, and so it is also pseudocompact.

Property $\left(1^{+}\right)$is quite strong; for example, it implies that the space is a $\Sigma$-space: just let $\mathcal{C}=\mathcal{F}$ be the countable family of $\left(1^{+}\right)$in the following definition:

Definition 3.7. A space $X$ is a $\Sigma$-space if it has a cover $\mathcal{C}$ by countably compact closed subsets and a $\sigma$-locally finite collection $\mathcal{F}$ of closed subsets such that, for every open $U \subset X$ and every $C \subset U, C \in \mathcal{C}$, there exists $F \in \mathcal{F}$ such that $C \subset F \subset U$.

$\Sigma$-spaces are a class of "generalized metric spaces" which have good preservation properties, some of which will be put to good use below.

Lemma 3.8. Let $X$ be a locally compact Hausdorff space. The countable closed discrete subspaces of $X$ form a P-ideal if, and only if, the extra point $\infty$ of the onepoint compactification $X+1$ of $X$ is an $\alpha_{1}$-point; that is, whenever $\left\{\sigma_{n}: n \in \omega\right\}$ is a countable family of sequences converging to $\infty$, then there is a sequence $\sigma$ converging to $\infty$ such that $\operatorname{ran}\left(\sigma_{n}\right) \subseteq^{*} \sigma$ for all $n$.

Proof. An ordinary sequence in $X$ converges to the extra point of $X+1$ if, and only if, its range is a closed discrete subspace of $X$.

A corollary of Theorem 2.5 and Lemmas 3.1 and 3.8 is that the extra point of $X+1$ is an $\alpha_{1}$-point whenever every countable subset of $X$ has Lindelöf closure. The converse is not true: for example, if $X$ is a separable, locally compact, countably compact, noncompact space, then the extra point of $X+1$ is vacuously $\alpha_{1}$.

The topic of $\alpha_{i}$-points $(i=1,1.5,2,3,4)$ in topological spaces has been a fruitful source of research ideas. See especially 20] and its references, where ZFC constructions are given of separable, locally compact, non-Lindelöf spaces $X$ such that the extra point of $X+1$ is an $\alpha_{2}$-point. It would therefore be interesting to know whether an " $\alpha_{1}$-point" can be weakened to an " $\alpha_{2}$-point" in the following theorem, even if Axiom $\mathcal{P}$ has to be replaced by some other axiom.

Theorem 3.9 (Axiom $\mathcal{P})$. Let $X$ be a locally compact Hausdorff space. Then at least one of the following is true:

$\left(1^{-}\right) X$ is the union of countably many closed pseudocompact subspaces.

(2) $X$ has an uncountable closed discrete subspace.

$\left(3^{+}\right)$The extra point of $X+1$ is not an $\alpha_{1}$-point.

Moreover, if $X$ has property $w D$, then item $\left(1^{-}\right)$can be strengthened to:

$\left(1^{\prime}\right) X$ is the union of countably many closed countably compact subspaces; in particular, $X$ is a $\Sigma$-space.

Proof. In the proof of Theorem 3.3, substitute the ideal $\mathcal{I}$ for $\mathcal{J}$ and $\mathcal{C C}$ for $\mathcal{P}$, and use the fact that if $\left(3^{+}\right)$fails, then $\mathcal{I}$ is a P-ideal. Stop before defining $A_{n}$ and just observe that $B_{n}$ is pseudocompact and hence has pseudocompact closure. For the "Moreover" part, use the fact that every pseudocompact space satisfying wD is countably compact [see the proof of Theorem 3.6]. 
As the notation suggests, (1) implies $\left(1^{-}\right)$: the closure of a pseudocompact subset is pseudocompact. Of course, $\left(1^{+}\right)$implies $\left(1^{\prime}\right)$. At the end of the following section we give two examples to show how $\left(1^{\prime}\right)$ cannot be replaced with $\left(1^{+}\right)$, nor $\left(1^{-}\right)$with (1), if one assumes $\mathrm{CH}$ or even MA.

Neither Theorem 3.3 nor Theorem 3.9 is a theorem of ZFC. In fact, a Souslin tree with the interval topology does not satisfy any of $\left(1^{-}\right),(2)$, or $(3)$ :

Example 3.10. Recall that a tree is a partially ordered set $T$ in which $\hat{t}=\{s \in T$ : $s \leq t\}$ is well ordered, and that a Souslin tree is an uncountable tree in which every chain (pairwise comparable subset) and antichain (pairwise incomparable subset) is countable. The interval topology on a tree is the one in which every point $p$ has a base of intervals of the form $(a, p]=\{t \in T: a<t \leq p\}$.

Now, every countable subset of a Souslin tree has countable closure, so (3) fails; every closed discrete subspace in a tree with the interval topology is a countable union of antichains [21, so (2) fails; and Souslin trees satisfy wD (in fact, every countable subset is contained in a countable clopen, hence metrizable subset). This last fact implies that $\left(1^{-}\right)$fails in the following way: every closed pseudocompact subset is countably compact, and every antichain is closed discrete; also, every countably compact uncountable subset of a tree contains an uncountable chain because its antichains are finite and because of the partition relation $\omega_{1} \rightarrow\left(\omega_{1}, \omega\right)^{2}$ [30.

The next application of our trichotomies is to normality in products. Recall that a space is called $\omega_{1}$-compact if every closed discrete subspace is countable. Clearly, every Lindelöf space and every countably compact space is $\omega_{1}$-compact.

Theorem 3.11 (Axiom $\mathcal{P}$ ). Let $X$ be a locally compact, normal, $\omega_{1}$-compact space. If the extra point of $X+1$ is an $\alpha_{1}$-point (in particular, if every countable subset of $X$ has Lindelöf closure in $X)$, then $X \times M$ is normal for all metrizable spaces $M$; in particular, $X$ is not a Dowker space.

Proof. Since neither (2) nor (3) of Theorem 3.9 applies, but $X$ satisfies wD (being normal), $\left(1^{+}\right)$applies, and we have a $\Sigma$-space. Every $\Sigma$-space is a Morita P-space; that is, the second player has a winning strategy in the following countable metacompactness game. On move $n$, Player I picks an open set $U_{n}$; then Player II plays a closed set $F_{n} \subset \bigcup_{i=1}^{n} U_{i}$. Player I wins if $X=\bigcup_{n=1}^{\infty} U_{n}$ and the $F_{n}$ do not cover $X$; otherwise Player II wins. A simple winning strategy for Player II is to choose $\mathcal{F}=\bigcup_{n} \mathcal{F}_{n}$ as in Theorem $\underline{3.6}$, with each $\mathcal{F}_{n}$ locally finite, and to let $F_{n}$ be the union of all the $C \in \mathcal{C}$ such that $C \subset F \subset \bigcup_{i=1}^{n} U_{i}$ for some $F \in \bigcup_{i=1}^{n} \mathcal{F}_{i}$.

As is well known [27, Corollary 4.1] normal Morita P-spaces are precisely those normal spaces whose product with every metrizable space is normal. As for the clause about "Dowker," one need only recall that a Dowker space is a normal space whose product with $[0,1]$ is not normal.

Definition 3.12. A space is [strongly] collectionwise Hausdorff if each closed discrete subspace has an expansion [see Definition 3.4 to a disjoint [resp. discrete] family of open sets.

We use the abbreviation "[s]cwH" for "[strongly] collectionwise Hausdorff". Obviously, every scwH space satisfies property wD.

Theorem 3.13 (Axiom $\mathcal{C C}+\mathrm{MA}\left(\omega_{1}\right)$ ). Every locally compact, hereditarily cwH Dowker space contains an uncountable closed discrete subspace. 
More generally, if a locally compact hereditarily cwH space $X$ is either countably tight or satisfies $w D$, then either $X$ is a $\Sigma$-space or $X$ has an uncountable closed discrete subspace.

Proof. It is easy to see that every hereditarily $\mathrm{cwH}$ space has the property that every separable subspace has countable spread; that is, every discrete subspace is countable. A celebrated theorem of Szentmiklóssy is that MA $\left(\omega_{1}\right)$ implies every locally compact Hausdorff space of countable spread is hereditarily Lindelöf 29. Hence (3) of Theorem 3.3 fails, so $\left(1^{+}\right)$fails, and so does (1) by the proof of Theorem 3.6 .

Corollary 3.14 (Axiom $\mathcal{C C}+\mathrm{MA}\left(\omega_{1}\right)$ ). If $X$ is locally compact, hereditarily cwH, $\omega_{1}$-compact and normal, then $X \times[0,1]$ is also normal.

Although this corollary is very specialized, it is actually one of the very few results to date of the form "It is consistent that there are no Dowker spaces that satisfy ..." and the only one besides Theorem 3.4 that uses local compactness in an essential way. In a future paper it will be shown that (unlike with Theorem 3.4) the large cardinal strength of Axiom $\mathcal{C C}$ is not needed for Corollary 3.14. However, some axioms beyond ZFC are required even for Corollary 3.14. I. Juhász constructed [17] an example of a locally compact Dowker space that was hereditarily separable (hence hereditarily cwH and $\omega_{1}$-compact) and even locally countable, using the axiom $\diamond$. Recently, one of us showed 24] that some versions of the Juhász construction yield an example which is, in addition, hereditarily normal $\left(\mathrm{T}_{5}\right)$ and has a one-point compactification whose extra point is $\alpha_{1}$. This last detail shows, thanks to Theorem 3.11, that some kinds of locally compact Dowker spaces need more than just $\mathrm{CH}$ for their construction.

\section{Embedding TheOREMS From $\alpha_{1}$ POINTS}

In this section we obtain some dichotomies using first $\mathcal{P}$ and then $P_{11}+C H$.

Definition 4.1. A space $X$ is an $\alpha_{1}$-space if every point of $X$ is an $\alpha_{1}$-point. A space $X$ is Fréchet-Urysohn if, whenever a point $x \in X$ is in the closure of $A \subset X$, then there is a sequence from $A$ converging to $x$. A v-space is a Fréchet-Urysohn $\alpha_{1}$-space.

If $Y$ is a compact Hausdorff space and $p$ is a point of $Y$, then $Y$ is naturally homeomorphic to the one-point compactification of $Y \backslash\{p\}$. By Lemma 3.8, a compact Hausdorff space is an $\alpha_{1}$-space if, and only if, the countable closed discrete subspaces of $Y \backslash\{p\}$ form a P-ideal for every point $p$ of $Y$. Adding the FréchetUrysohn property makes for quite a sharp result:

Theorem 4.2 (Axiom $\mathcal{P}$ ). If $Y$ is a locally compact Hausdorff $v$-space, then $Y$ is either first countable or contains the one-point compactification of an uncountable discrete space.

Proof. It clearly suffices to show this for the compact case. Let $p \in Y$, and let $X=Y \backslash\{p\}$. Condition $\left(3^{+}\right)$of Theorem 3.9 does not hold, and if (2) holds for $X$, then the union of $\{p\}$ with the uncountable closed discrete subspace given by (2) is as desired. If neither $\left(3^{+}\right)$nor (2) holds, then alternative (ii) of Axiom $\mathcal{P}$ comes into play, giving us subsets $B_{n}$ covering $X$, such that every countable subset of each $B_{n}$ has an accumulation point somewhere in $X$. By the Fréchet-Urysohn 
property, $p$ cannot be in the closure of any $B_{n}$ in $Y$. Hence each $B_{n}$ has compact closure in $X$, making $p$ a $G_{\delta}$-point and hence a point of first countability in the compact Hausdorff space $Y$.

The foregoing proof also provides a proof of the following more general fact:

Theorem 4.3 (Axiom $\mathcal{P})$. Let $Y$ be a locally compact Hausdorff Fréchet-Urysohn space. Every $\alpha_{1}$-point of $Y$ is either of countable character or is the one relatively nonisolated point in a subspace $E$ of $Y$ such that $E$ is the one-point compactification of an uncountable discrete space.

In the following analogue of Theorem 4.3, "Fréchet-Urysohn" is weakened to "countably tight" while the $\alpha_{1}$-condition gets strenghtened:

Theorem 4.4 (Axiom $\mathcal{C C}$ ). Let $Y$ be a locally compact Hausdorff space and let $p$ be a point of $Y$ with a countably tight neighborhood. If every countable subset of $Y \backslash\{p\}$ has Lindelöf closure there, then either $p$ is of countable character or it is the one relatively nonisolated point in a subspace $E$ of $Y$ such that $E$ is the one-point compactification of an uncountable discrete space.

Proof. In $X=Y \backslash\{p\}$, alternative (3) of Theorem 3.3 fails. If (2) then holds, we are done as in the proof of Theorem 4.2. otherwise, let $N$ be a countably tight compact neighborhood of $p$ in $Y$. Compact spaces of countable tightness are precisely those in which there is no perfect preimage of $\omega_{1}$ (an easy consequence of Lemma 1 in [11), so (1a) applies to $N \backslash\{p\}$. This implies that $p$ is a point of first countability as in the proof of Theorem 4.2

The one-point compactification of a Souslin tree shows that neither Theorem 4.2 nor Theorem 4.3 is a theorem of ZFC. It is a $v$-space in which the extra point is not a point of first countability, yet every countable subset has countable closure, and there is no one-point compactification of an uncountable discrete space anywhere in the space.

We will now show how these applications can be done without assuming large cardinal axioms, by substituting the combination of $P_{11}$ and $\mathrm{CH}$ for $\mathcal{P}$. We first take care of spaces of character $\alpha_{1}$ and then use $\mathrm{CH}$ to remove the character condition. [Recall that a space is of character $\leq \kappa$ if every point has a base of cardinality $\leq \kappa$ for its neighborhoods.]

Theorem 4.5 (Axiom $P_{11}$ ). If $Y$ is a locally compact Hausdorff v-space of character $\alpha_{1}$, then $Y$ contains the one-point compactification of an uncountable discrete space.

Proof. Let $p$ be of character $\alpha_{1}$, and let $\left\{V_{\alpha}: \alpha<\omega_{1}\right\}$ be a family of closed neighborhoods of $p$ in $Y$ whose intersection is $\{p\}$. We will use the well-known easy fact that if a family of closed neighborhoods of a point $z$ in a compact Hausdorff space intersects in $\{z\}$, then the collection of finite intersections of members of this family forms a local base at $z$.

For each countable ordinal $\alpha$, let $K_{\alpha}=\bigcap\left\{V_{\beta}: \beta<\alpha\right\}$. Since $p$ is of uncountable character, $K_{\alpha} \backslash\{p\} \neq \emptyset$ for all countable $\alpha$. Let $Z=\left\{z_{\alpha}: \alpha<\omega_{1}\right\}$ be such that $z_{\alpha} \in K_{\alpha} \backslash\{p\}$. Since $\bigcap\left\{V_{\beta}: \beta<\alpha\right\}=\{p\}, Z$ is uncountable; also, each neighborhood of $p$ contains all but countably many points of $Z$. Each point of $X=Y \backslash\{p\}$ has a neighborhood meeting at most countably many points of $Z$. 
Now $X$ is locally compact and its family of closed discrete subspaces is a $\mathrm{P}$ ideal, as is the family of discrete subspaces of $Z$ that are closed in $X$. Thus $P_{11}$ implies that either there is an uncountable subset $A$ of $Z$ such that every countable subset is in the latter P-ideal, or there is an uncountable subset $B$ of $Z$ such that no infinite subset of $B$ is closed discrete in $X$. However, this second alternative is impossible: $B$ would have $p$ in its closure, and yet there would be no sequence converging from $B$ to $p$, violating the Fréchet-Urysohn property. Thus the first alternative holds, and it implies that $A$ is a closed discrete subspace of $X$, because $X$ is Fréchet-Urysohn. This makes $A \cup\{p\}$ the one-point compactification of an uncountable discrete space.

Theorem 4.6 $\left(P_{11}+C H\right)$. If $Y$ is a locally compact Hausdorff $v$-space, then $Y$ is either first countable or contains the one-point compactification of an uncountable discrete space.

Proof. It clearly suffices to show this for the compact case. In 18, it is shown that $\mathrm{CH}$ implies that any point $p$ of uncountable character in a compact Hausdorff countably tight space $K$ is the limit of a convergent $\omega_{1}$-sequence from $K \backslash\{p\}$. If we let $Z$ be the range of this sequence, we can just follow the last paragraph of the preceding proof, keeping in mind that every point of $X=K \backslash\{p\}$ has a neighborhood meeting at most countably many points of $Z$.

The foregoing proof also shows that Theorem 4.3 continues to hold if $P_{11}+\mathrm{CH}$ is substituted for $\mathcal{P}$. Omitting $\mathrm{CH}$ from the set-theoretic hypotheses, we get the same theorem for $\alpha_{1}$-points of character exactly $\aleph_{1}$.

We close this section with two examples promised earlier, showing why the various trichotomies used different variations on (1), (2), and (3) of Theorem 3.3.

Example 4.7. Using the axiom $\mathfrak{b}=\mathfrak{c}$, E. K. van Douwen [5] (Theorem 13.4) constructed a locally compact, locally countable, countably compact Hausdorff space $X$ which admits a closed map onto $[0,1]$. If $X=\bigcup_{n=0}^{\infty} X_{n}$, then the projection of at least one $X_{n}$ must have some open interval in its closure. Then if $Q$ is a countable subset of $X_{n}$ which projects to a dense subset of this interval, the closure of $Q$ in $X$ is uncountable and hence non-Lindelöf. Thus (1) does not hold even though $\left(1^{\prime}\right)$ holds trivially. Also, $Q$ witnesses that (3) of Theorem 3.3 holds, but $\left(3^{+}\right)$of Theorem 3.9 does not, because $X$ is countably compact and so $X+1$ is trivially $\alpha_{1}$. Of course, (2) also fails.

The following example also shows that "Fréchet-Urysohn" cannot be weakened to "sequential" in Theorems 4.2 and 4.3 nor even to "of sequential order 2".

Example 4.8. Using $\mathrm{CH}$, Jakovlev constructed [16] a space $X$ with underlying set $\omega_{1} \times \omega$ that is locally compact, locally countable, Hausdorff, and pseudocompact; in fact, $W_{n}=\omega_{1} \times n$ is open and dense in $X$ for every $n \in \omega$, and every infinite subset of $W_{n}$ has a limit point in $W_{n+1}$. This easily implies that $X$ is not countably metacompact and that (2) of Theorems 3.3 and 3.9 does not hold. Neither does (1) of Theorem 3.3 nor $\left(1^{\prime}\right)$ of 3.9 any set $D$ that meets each row $\omega_{1} \times\{n\}$ in at most a singleton is clearly closed discrete, but it is easy to see from the above that the closure of every uncountable set contains an infinite subset $D$ like this, and hence cannot be countably compact. Also, $\omega \times\{0\}$ has uncountable (hence, by local countability, non-Lindelöf) closure, so that (3) of Theorem 3.3 does hold. 
However, $\left(3^{+}\right)$of Theorem 3.9 does not hold: the extra point $\infty$ of $X+1$ is not only an $\alpha_{1}$-point, but it also has a weak base consisting of the sets $(X+1) \backslash W_{n}$. This means that a set is an open neighborhood of $\infty$ if, and only if, it meets $X$ in an open set and also contains one of the sets $(X+1) \backslash W_{n}$. From this follows not only the fact that $X+1$ is $\alpha_{1}$, but also that it is sequential of order 2 ; in fact, a set $F$ that is closed in $X$ is either compact or meets every set of the form $(X+1) \backslash W_{n}$.

This was the first example under any hypotheses of a weakly first countable compact Hausdorff space which is not first countable, and it is still unsolved whether the existence of such a space follows just from ZFC. However, the construction is easily adapted to produce a similar space under MA or even under $\mathfrak{b}=\mathfrak{c}$, with an underlying set $\mathfrak{c} \times \omega$, having all the topological properties listed above for Jakovlev's space. Thus there seems little hope of replacing $\left(1^{-}\right)$with (1) in Theorem 3.9 in any model of $\mathcal{P}$ or even of $\mathcal{C C}$.

\section{Applications of $C C_{12}$ to Manifolds}

One of the most powerful recent applications of the axioms studied here is the main theorem of [25]:

Theorem 5.1 (PFA). Every hereditarily normal, hereditarily cwH manifold of dimension $>1$ is metrizable.

A manifold is a Hausdorff space in which every point has a neighborhood homeomorphic to $\mathbb{R}^{n}$ for some $n \in \omega$. The topological statement in this theorem is a strengthening of the celebrated theorem of Mary Ellen Rudin [31] of the consistency of every perfectly normal manifold being metrizable. [Rudin showed that this is implied by MA $+\neg \mathrm{CH}$, which follows from the PFA.] Indeed, every perfectly normal space is hereditarily normal, every perfectly normal manifold is hereditarily $\mathrm{cwH}$ [33], 222, and the only nonmetrizable manifolds of dimension 1 (the long ray and long line) are not perfectly normal.

Theorem 5.1 made heavy use of $C C_{22}$. It also used an application of $C C_{12}$ in 23 whose proof appears here for the first time (Theorem 5.8 below). We will also use this applicaction to eliminate certain kinds of perfectly normal manifolds even if $\mathrm{CH}$ is assumed:

Theorem $5.13\left(C C_{12}\right)$. Let $M$ be a perfectly normal manifold. Then either $M$ is metrizable, or it contains a countable subset with nonmetrizable closure.

Some such dichotomy is the best that can be hoped for in the way of CHcompatible results: in 1976, Rudin and Zenor were able to construct a perfectly normal nonmetrizable manifold of dimension 2 using $\mathrm{CH}$ alone 33. Rudin has also used stronger axioms to construct perfectly normal manifolds that cannot be obtained from $\mathrm{CH}$ alone. For example, she showed that $\diamond$ is enough to construct perfectly normal, countably compact noncompact manifolds [22, while one of us has shown [6] that $\mathrm{CH}$ is compatible with the statement that perfectly normal countably compact spaces are compact, and so the manifolds among them are metrizable. Theorem 5.13 shows that another example of Rudin requires something more than just $\mathrm{CH}$ : with the help of the axiom $\diamond^{+}$, Rudin constructed a perfectly normal nonmetrizable manifold in which every countable subset has metrizable closure [32. 
One ingredient in the proof of Theorem 5.13 is the fact that if every countable subset of a manifold $M$ has metrizable closure, then $M$ is a topological direct sum of so-called Type I spaces:

Definition 5.2. A space $X$ is a Type $I$ space if it is the union of an ascending $\omega_{1}$-sequence $\left\langle X_{\alpha}: \alpha<\omega_{1}\right\rangle$ of open subspaces such that $\bar{X}_{\alpha} \subset X_{\beta}$ whenever $\alpha<\beta$ and such that $\bar{X}_{\alpha}$ is Lindelöf for all $\alpha$.

Examples of Type I spaces include Aronszajn trees and many important manifolds [22. Every Type I space clearly has the property that every Lindelöf [in particular, every countable] subset is contained in some $X_{\alpha}$ and hence has Lindelöf closure.

Definition 5.3. Given a faithfully indexed subset $D=\left\{d_{\alpha}: \alpha \in A\right\}$ of a set $X$, an expansion of $D$ is a family $\left\{U_{\alpha}: \alpha \in A\right\}$ such that $U_{\alpha} \cap D=d_{\alpha}$ for all $\alpha \in A$.

A space $X$ is [strongly] collectionwise Hausdorff (often abbreviated [s]cwH) if every closed discrete subspace expands to a disjoint [resp. discrete] collection of open sets.

The following is well known; cf. [10].

Lemma 5.4. Every locally Lindelöf, paracompact space is the topological direct sum of Lindelöf subspaces.

Definition 5.5. Let $X$ be a Type I space. A sequence $\left\langle X_{\alpha}: \alpha<\omega_{1}\right\rangle$ as in Definition 5.2 is called a canonical sequence for $X$ if $X_{\gamma}=\bigcup\left\{X_{\alpha}: \alpha<\gamma\right\}$ for each limit ordinal $\gamma$. Given a canonical sequence $\Sigma=\left\langle X_{\alpha}: \alpha<\omega_{1}\right\rangle$ for $X$, the skeleton of $X$ associated with $\Sigma$ is the sequence $\left\langle\bar{X}_{\alpha} \backslash X_{\alpha}: \alpha<\omega_{1}\right\rangle$. The set $\bar{X}_{\alpha} \backslash X_{\alpha}$ is known as the $\alpha$ th bone of $X$ associated with $\Sigma$.

A basic result about Type I spaces is that any pair of canonical sequences agree on a set of terms indexed by a club. Hence any two skeletons agree on a club set of bones; see Lemma 4.4 of [22]. The following slightly generalizes Lemma 4.10 of 22.

Theorem 5.6. Let $X$ be a Type I space. The following are equivalent:

(i) $X$ is paracompact.

(ii) Some skeleton of $X$ is empty.

(iii) For every skeleton of $X$, there is a club $C \subset \omega_{1}$ such that the bones indexed by $C$ are empty.

Proof. (i) if and only if (ii): If $X$ is paracompact, then by Lemma 5.4, $X$ is a topological direct sum of Lindelöf subspaces. Now, every open cover of a Type I space has a subcover of cardinality $\leq \aleph_{1}$, and so there can be at most $\aleph_{1}$ summands. Listing them in order type $\omega_{1}$ [with repetitions if there are only countably many] and letting $X_{\alpha}$ be the union of the summands indexed by ordinals $<\alpha$, we arrive at a canonical sequence with an empty skeleton. The reverse implication is trivial.

(ii) if and only if (iii): This is immediate from the fact that any two canonical sequences agree on a club set of indices.

The proof of Lemma 4.11 in [22] also shows the following theorem, in which the key to necessity is the Pressing-Down Lemma:

Theorem 5.7. Let $X$ be a locally ccc Type I space. Then $X$ is cwH if, and only if, every closed discrete subspace of $X$ misses the bones indexed by a club. 
The interplay between these last two theorems begins with the following theorem (Theorem $\mathrm{C}$ of [23], used there and in [24], with its proof promised for this paper).

Theorem $5.8\left(C C_{12}\right)$. Let $X$ be a locally compact, cwH, Type I space. If $X$ is either strongly $c w H$ or locally ccc, then either:

(1) $X$ is paracompact, or

(2) $X$ contains a perfect preimage of $\omega_{1}$ that is closed in $X$.

Proof. Suppose (1) fails. Let $\Sigma=\left\langle X_{\alpha}: \alpha<\omega_{1}\right\rangle$ be a canonical sequence for $X$. There is a stationary set $S$ such that the bones indexed by $S$ are nonempty. For each $\alpha \in S$, let $x_{\alpha}$ be chosen from the $\alpha$ th bone of $X$. If we let $W=\left\{x_{\alpha}: \alpha \in S\right\}$, then every point of $X$ has a neighborhood meeting $W$ in at most a countable set.

Let $\mathcal{J}$ be the ideal of all countable subsets of $W$ with compact closure in $X$. As in Lemma 3.1, $\mathcal{J}$ is countable-covering, and $\mathcal{J}^{\perp}$ is the ideal of all countable subsets of $W$ that are closed discrete in $X$. By $C C_{12}$ applied to $\mathcal{J}$, there is either (a) an uncountable subset $W^{\prime}$ of $W$ such that every countable subset of $W^{\prime}$ has compact closure in $X$, or (b) a discrete subspace of $W$ that is not only closed in $X$ but also of the form $\left\{x_{\alpha}: \alpha \in S^{\prime}\right\}$, where $S^{\prime}$ is a stationary subset of $S$.

Now (b) cannot happen if $X$ is locally ccc because of Theorem 5.7 If $X$ is not locally ccc, and (b) holds, then every discrete family of open subsets of any $\overline{X_{\gamma}}$ must be countable because $\overline{X_{\gamma}}$ is Lindelöf. Now, the cwH property gives a disjoint open expansion $\left\{G_{\alpha}: \alpha \in S^{\prime}\right\}$ of $\left\{x_{\alpha}: \alpha \in S^{\prime}\right\}$, and the Pressing-Down Lemma implies that there is a stationary subset $S^{\prime \prime}$ of $S^{\prime}$ and $\gamma<\omega_{1}$ such that $G_{\alpha} \cap X_{\gamma} \neq \emptyset$ for all $\alpha \in S^{\prime \prime}$. Hence the expansion cannot be discrete, and $X$ cannot be strongly cwH.

So (a) must hold. Now $C=c \ell\left(W^{\prime}\right)$ is $\omega$-bounded and noncompact: clearly $\left\{X_{\alpha}: \alpha<\omega_{1}\right\}$ is an open cover of $C$ without a countable subcover; and if $Q$ is a countable subset of $C$, then $Q$ is contained in some $X_{\alpha}$ and is in the closure of $\left\{x_{\beta}: \beta<\alpha\right.$ and $\left.\beta \in W^{\prime}\right\}$, so $Q$ has compact closure in $X$.

Next, list $W^{\prime}$ in natural order as $\left\{y_{\alpha}: \alpha<\omega_{1}\right\}$ and, for each $\alpha \in \omega_{1}$, let $C_{\alpha}=$ $c \ell\left(\left\{y_{\xi}: \xi<\alpha\right\}\right) \backslash X_{\nu(\alpha)}$ where $\nu(\alpha)$ is the least ordinal $\eta$ such that $\left\{y_{\xi}: \xi<\alpha\right\} \subset$ $X_{\eta}$. Then $C_{\alpha}$ is compact for all $\alpha$ and the natural map $f: \bigcup\left\{C_{\alpha}: \alpha \in \omega_{1}\right\} \rightarrow \omega_{1}$ is easily seen to be closed and continuous, hence perfect. The domain of $f$ is closed since $X_{\nu(\alpha)} \cap\left\{y_{\xi}: \xi<\alpha\right\}$ has compact closure in $X$ for all $\alpha$.

The two possible conclusions in the preceding theorem (as well as in all results of this section and Section 4, where we present two alternative conclusions) are easily seen to be mutually exclusive in ZFC. It is for the sake of brevity that we write "either ... or" rather than "exactly one of the following is true".

The following lemma dovetails with Theorem 5.8 by giving a natural internal criterion for partitionability of some spaces into clopen Type I subspaces. As part of the proof, we also give an alternative characterization of Type I spaces.

Lemma 5.9. Let $X$ be a locally compact space. If either (a) $X$ is locally connected and countably tight or (b) $X$ is perfectly normal and collectionwise Hausdorff, then the following are equivalent:

(i) $X$ is a topological direct sum of Type I spaces.

(ii) Every Lindelöf subset of $X$ has Lindelöf closure. 
Proof. If ( $i$ ) is true, then every Lindelöf subset of $X$ meets at most countably many summands. In each of these summands, its overlap with the summand is contained in a closed Lindelöf subspace. Hence (i) implies (ii).

To show the converse, we first assume $(a)$ is true. Since $X$ is locally connected, it is the topological direct sum of its components. So we need only show each component is of Type I. Beginning with any point $x$, we build up a sequence of $\sigma$ compact open subspaces $\left\{U_{\alpha}: \alpha<\omega_{1}\right\}$ containing $x$ such that $\bar{U}_{\alpha} \subset U_{\beta}$ whenever $\alpha<\beta$. Let $U_{0}$ be any open neighborhood of $x$ with compact closure. If $U_{\xi}$ has Lindelöf closure and $\alpha=\xi+1$, then we cover the closure of $U_{\xi}$ with countably many open sets with compact closures, and let $U_{\alpha}$ be their union. The union of the closures is $\sigma$-compact, so it (and hence $U_{\alpha}$ itself) has Lindelöf closure.

If $\alpha$ is a countable limit ordinal and $U_{\xi}$ has been defined for $\xi<\alpha$, we let $U_{\alpha}$ be the union of the earlier $U_{\xi}$. By the obvious induction hypothesis, $U_{\alpha}$ is $\sigma$-compact and hence has Lindelöf closure. The (open, Type I) space $\bigcup\left\{U_{\alpha}: \alpha<\omega_{1}\right\}$ is also closed because $X$ is countably tight, and hence it contains the component of $x$ in $X$. But any clopen subset of a Type I space is of Type I.

If (b) holds, we recall a definition and a theorem of Gruenhage. A space is said to have Lindelöf number $<\kappa$ if every open cover has a subcover of cardinality $<\kappa$. Gruenhage has shown 12 that every locally compact, cwH, perfectly normal space is the topological direct sum of subspaces of Lindelöf number $\leq \alpha_{1}$. We will thus be done as soon as we show:

Claim. A space is of Type I if, and only if, it is locally Lindelöf, has Lindelöf number $\leq \alpha_{1}$, and has the property that every Lindelöf subset has Lindelöf closure.

Proof of the Claim. Necessity is clear. To show sufficiency, we first cover $X$ with open subsets $\left\{G_{\alpha}: \alpha<\omega_{1}\right\}$ such that each $G_{\alpha}$ has Lindelöf closure, using the easy fact that each point has an open neighborhood with Lindelöf closure. Let $U_{0}=G_{0}$ and follow the construction of $\left\{U_{\alpha}: \alpha<\omega_{1}\right\}$ above, with the added requirement that $G_{\alpha} \subset U_{\beta}$ whenever $\alpha<\beta$.

The following is immediate from Theorem 5.8 and Lemma 5.9,

Theorem 5.10 $\left(C C_{12}\right)$. Let $X$ be locally compact, locally connected, and countably tight. If $X$ is either strongly cwH or locally ccc and cwH, and every Lindelöf subset of $X$ has Lindelöf closure, then either:

(1) $X$ is paracompact, or

(2) X has a closed subspace which is a perfect preimage of $\omega_{1}$.

Theorem $5.11\left(C C_{12}\right)$. Let $X$ be a locally compact, perfectly normal, collectionwise Hausdorff space. If every Lindelöf subspace of $X$ has Lindelöf closure, then $X$ is paracompact.

Proof. Since $X$ is normal and cwH, it is strongly cwH. Since it is locally compact and perfectly normal, it is countably tight. So it is clear from Theorem 5.8 and Lemma 5.9 that $X$ is either paracompact or contains a perfect preimage of $\omega_{1}$. However, no perfectly normal space can contain a perfect preimage of $\omega_{1}$ : the set of fibers over the limit ordinals is not a $G_{\delta}$ because of the Pressing-Down Lemma.

Corollary $5.12\left(C C_{12}\right)$. Let $X$ be locally compact, locally connected, and perfectly normal. If every Lindelöf subset of $X$ has Lindelöf closure, then $X$ is paracompact. 
Proof. Perfectly normal, locally compact, locally connected spaces are collectionwise Hausdorff (cf. [22, Corollary 2.17]), and so this is immediate from Theorem 5.11 .

Theorem $5.13\left(C C_{12}\right)$. Let $M$ be a perfectly normal manifold. Then $M$ is either metrizable or contains a countable subset with nonmetrizable closure.

Proof. Manifolds are locally compact and locally connected, and it is clear from Definition 5.3 and Urysohn's metrization theorem that every paracompact manifold is metrizable. Also, from the elementary fact that separability, second countability, and Lindelöfness are all equivalent for metrizable spaces, we get the equivalence of "every countable subset has metrizable closure" and "every separable subset has Lindelöf closure". Since every point in a manifold has a separable metrizable neighborhood, the latter is in turn equivalent to "every Lindelöf subset has Lindelöf closure."

In view of this theorem, it is hardly surprising that the main example of [33] is separable. Another example in [33, whose construction was only outlined, was not separable; however, it too had a separable nonmetrizable subspace, and Theorem 5.13 shows this was unavoidable for a " $\mathrm{CH}$ alone" example.

Theorem $5.14\left(C C_{12}\right)$. Let $M$ be a collectionwise Hausdorff manifold in which every countable subset has metrizable (equivalently, Lindelöf) closure. Then either

(1) $M$ contains a perfect preimage of $\omega_{1}$ or

(2) $M$ is metrizable.

Proof. As for Theorem 5.13, using Theorems 5.8 and 5.10 in place of Corollary 5.12 .

We cannot strengthen "perfect preimage of $\omega_{1}$ " to "copy of $\omega_{1}$ " in any of the theorems in this section; the club-guessing axiom $\boldsymbol{\phi}_{C}$ is enough to imply the existence of a hereditarily collectionwise normal 2-manifold which is a perfect preimage of $\omega_{1}$ but contains no copy of $\omega_{1}$ [26]. This also shows that Theorem 5.1 required something in addition to $M A+\neg C H+C C_{22}$.

\section{Consistency proofs}

Here at last we will prove that the statement $P_{22}$ is consistent with $\mathrm{ZFC}+\mathrm{CH}$; since the proof is a modification of that of Abraham and Todorcevic $\underline{3}$, we will include only the new ideas and refer the reader to 3 for some of the details. The papers 9 and [6] might also prove helpful for some of the basics of totally proper forcing.

Definition 6.1. Let $P$ be a notion of forcing, and let $N$ be a countable elementary submodel of $H(\lambda)$ for some large enough regular $\lambda$. We say a condition $q \in P$ is totally $(N, P)$-generic if whenever $D$ is a dense open subset of $P$ that is in $N$, we can find a condition $p \in N \cap D$ with $q \leq p$. We say that $P$ is totally proper if, given $N$ as above, every $p \in N \cap P$ has a totally $(N, P)$-generic extension $q$.

Clearly totally proper forcings are proper, and it is not hard to see that a notion of forcing is totally proper if and only if it is proper and forcing with it adds no new countable sequences of elements from the ground model (so in particular, totally proper forcings add no new reals to the ground model). 
Let us begin by assuming that the Continuum Hypothesis holds and that $\mathcal{I}$ is a $P$-ideal in $\left[\omega_{1}\right]^{\aleph_{0}}$. Furthermore, let $\left\langle A_{\alpha}: \alpha<\omega_{1}\right\rangle$ be a generating sequence for $\mathcal{I}$; i.e.,

- $A_{\alpha} \subseteq \alpha$,

- $\alpha<\beta \Longrightarrow A_{\alpha} \subseteq^{*} A_{\beta}$, and

- if $A \in \mathcal{I}$, then $A \subseteq A_{\alpha}$ for some $\alpha$.

Our assumption that $\mathrm{CH}$ holds guarantees that such a sequence can be found.

Assume that for this particular $P$-ideal there is no stationary set $S$ such that $[S]^{\aleph_{0}} \cap \mathcal{I}=\emptyset$, i.e., the second alternative of Axiom $P_{22}$ fails. We will define a totally proper notion of forcing $P$ that will adjoin a stationary set $S$ witnessing that the first alternative of Axiom $P_{22}$ must hold; i.e., every initial segment of $S$ is in the ideal $\mathcal{I}$.

Definition 6.2. A function $f$ is called a promise if

(1) $\operatorname{dom} f$ is a stationary subset of $\omega_{1}$, and

(2) for each $\alpha \in \operatorname{dom} f, f(\alpha)$ is a finite subset of $A_{\alpha}$.

Definition 6.3. A condition $p \in P$ consists of a pair $\left(x_{p}, \Phi_{p}\right)$, where

(1) $x_{p}: \alpha \rightarrow 2$ for some $\alpha<\omega_{1}$,

(2) $x_{p}^{-1}(\{1\})$ (henceforth denoted $[p]$ or $\left[x_{p}\right]$ ) is in $\mathcal{I}$,

(3) $\Phi_{p}$ is a countable set of promises.

A condition $q$ extends $p$ (written $q \leq p$ ) if

(4) $x_{q} \supseteq x_{p}$,

(5) $\Phi_{q} \supseteq \Phi_{p}$,

(6) for every $f \in \Phi_{p}$, the set

$$
Y(f, q, p):=\left\{\alpha \in \operatorname{dom} f:[q] \backslash[p] \subseteq A_{\alpha} \backslash f(\alpha)\right\}
$$

is stationary, and $f \uparrow Y(f, q, p) \in \Phi_{q}$.

For $p \in P$, the set $[p]$ is the approximation to the stationary set we are trying to adjoin, while $\Phi_{p}$ puts some constraints on how our approximation is allowed to grow. The reader is invited to show that $P$ is in fact a partially ordered set transitivity follows from the last clause in the definition of $q \leq p$.

The next proposition will come in handy in places where we need to ensure that a certain decreasing sequence of conditions in $P$ has a lower bound.

Proposition 6.4. For every $\delta<\omega_{1}, p \in P$, and finite $F$, Player I has a winning strategy in the following game:

Stage 0: $F_{0}=F, p_{0}=p$.

Stage $n+1$ : Player I choose a finite $F_{n+1} \supseteq F_{n}$, and Player II responds by choosing $p_{n+1} \leq p_{n}$ with $\left[p_{n+1}\right] \backslash\left[p_{n}\right] \subseteq A_{\delta} \backslash F_{n+1}$.

After $\omega$ stages, Player I wins if $\left\{p_{n}: n \in \omega\right\}$ has a lower bound $q$ such that

$$
[q]=\bigcup_{n \in \omega}\left[p_{n}\right]
$$

Proof. We put ourselves in the role of Player I, and consider Player II to be the opponent. Note that no matter which strategy we use, it will always be the case that

$$
\bigcup_{n \in \omega}\left[p_{n}\right] \subseteq A_{\delta} \cup[p] \in \mathcal{I}
$$


and so the existence of a lower bound will hinge on the promises involved. A quick look at the definitions involved shows us that we must ensure for all $i \in \omega$ and $f \in \Phi_{p_{i}}$ that

$$
K(f, i):=\left\{\xi \in \operatorname{dom} f: \bigcup_{n \in \omega}\left[p_{n}\right] \backslash\left[p_{i}\right] \subseteq A_{\xi} \backslash f(\xi)\right\} \text { is stationary, }
$$

as then

$$
q:=\left(\bigcup_{n \in \omega}\left[p_{n}\right], \bigcup_{n \in \omega} \Phi_{p_{n}} \cup \bigcup\left\{f \uparrow K(f, n): f \in \Phi_{p_{n}}\right\}\right)
$$

will be the desired lower bound.

We fix some bookkeeping procedure that at stage $n+1$ of the game will hand us an $f \in \Phi_{p_{i}}$ for some $i \leq n$, and such that every promise appearing in some $\Phi_{p_{i}}$ is eventually treated in this way.

At stage $n+1$, we will be handed $p_{n}$ and $f \in \Phi_{p_{i}}$ for some $i \leq n$. Since $Y\left(f, p_{n}, p_{i}\right)$ is stationary and $A_{\delta} \subseteq^{*} A_{\alpha}$ for all $\alpha>\delta$, there is a finite $\overline{\bar{F}}_{n}$ such that

$$
K(f, n, i):=\left\{\xi \in Y\left(f, p_{n}, p_{i}\right): A_{\delta} \backslash \bar{F} \subseteq A_{\xi} \backslash f(\xi)\right\} \text { is stationary. }
$$

We set $F_{n+1}=F_{n} \cup \bar{F}_{n}$.

This finishes the description of our strategy; we must verify that it ensures (6.3) for every relevant promise $f$. Given $i \in \omega$ and $f \in \Phi_{p_{i}}$, we choose $n \geq i$ so that our bookkeeping hands us $f$ at stage $n+1$. Letting $[q]$ denote $\bigcup_{n \in \omega}\left[p_{n}\right]$, we note that $[q] \backslash\left[p_{i}\right]$ is equal to $[q] \backslash\left[p_{n}\right] \cup\left[p_{n}\right] \backslash\left[p_{i}\right]$, and therefore

$$
[q] \backslash\left[p_{i}\right] \subseteq A_{\delta} \backslash F_{n+1} \cup\left[p_{n}\right] \backslash\left[p_{i}\right] \subseteq A_{\delta} \backslash \bar{F}_{n} \cup\left[p_{n}\right] \backslash\left[p_{i}\right] .
$$

As a consequence, we see

$$
K(f, n, i) \subseteq\left\{\xi \in \operatorname{dom} f:[q] \backslash\left[p_{i}\right] \subseteq A_{\xi} \backslash f(\xi)\right\}=K(f, i),
$$

and therefore (6.3) is established.

Definition 6.5. If $p \in P$ and $D \subseteq P$ is dense, we say that $\alpha<\omega_{1}$ is bad for $p$ and $D$ if there is a finite set $F_{\alpha} \subseteq A_{\alpha}$ such that for no $q \leq p$ do we have $q \in D$ and $[q] \backslash[p] \subseteq A_{\alpha} \backslash F_{\alpha}$. We let $\operatorname{Bad}(p, D)$ consist of all $\alpha<\omega_{1}$ that are bad for $p$ and $D$.

Lemma 6.6. If $p \in P$ and $D \subseteq P$ is dense, then $\operatorname{Bad}(p, D)$ is nonstationary.

Proof. Suppose this is not the case. The function $f$ with domain $\operatorname{Bad}(p, D)$ that sends $\alpha$ to $F_{\alpha}$ (from the previous definition) is then a promise. Let $r:=\left(x_{p}, \Phi_{p} \cup\right.$ $\{f\})$; clearly $r$ is an extension of $p$ in $P$. Since $D$ is dense, there is $q \leq r$ with $q \in D$. Now we know that $Y(f, q, r)$ is stationary, and if $\alpha \in Y(f, q, r)$, then

$$
[q] \backslash[r]=[q] \backslash[p] \subseteq A_{\alpha} \backslash f(\alpha)=A_{\alpha} \backslash F_{\alpha},
$$

and this contradicts the choice of $F_{\alpha}$.

Definition 6.7. If $f$ is a promise, then we say $\alpha<\omega_{1}$ is banned by $f$ if the set of $\xi \in \operatorname{dom} f$ with $\alpha \in A_{\xi} \backslash f(\xi)$ is nonstationary. The set $\operatorname{Ban}(f)$ consists of all those $\alpha<\omega_{1}$ that are banned by $f$.

Lemma 6.8. If $f$ is a promise, then $\operatorname{Ban}(f)$ is nonstationary. 
Proof. Suppose this is not the case. By our assumption on $\mathcal{I}$ we know

$$
[\operatorname{Ban}(f)]^{\aleph_{0}} \cap \mathcal{I} \neq \emptyset
$$

so let $A$ witness this. Because $A \in \mathcal{I}$, there is some $\alpha$ such that $A \subseteq{ }^{*} A_{\alpha}$. Note that this holds for all large $\beta$ as well. Thus for each $\beta>\alpha$ in $\operatorname{dom}(f)$, we can fix a finite $F_{\beta}$ such that

$$
A \backslash F_{\beta} \subseteq A_{\beta} \backslash f(\beta) .
$$

We can then find a single finite $F \subseteq A$ so that the set of $\beta \in \operatorname{dom}(f)$ with $A \backslash F \subseteq$ $A_{\beta} \backslash f(\beta)$ is stationary. We have a contradiction now, as $A \backslash F$ is a nonempty subset of $\operatorname{Ban}(f)$.

Theorem 6.9. The forcing $P$ is totally proper. Moreover, if $\dot{S}$ is a $P$-name for $\bigcup\left\{[p]: p \in \dot{G}_{P}\right\}$, then every condition forces $\dot{S}$ to be a stationary subset of $\omega_{1}$ with every countable subset in $\mathcal{I}$.

Proof. Suppose $p \in P$, and let $N \prec H(\lambda)$ be a countable model containing $p, P$, $\dot{S}$, and the generating sequence for $\mathcal{I}$. Let $\delta=N \cap \omega_{1}$. We first prove that there is a totally $(N, P)$-generic $q \leq p$ with $\delta \in[q]$.

Our construction of $q$ uses arguments from [3], with a bit of care taken so that we can ensure that $\delta \in[q]$. Letting $\left\{D_{n}: n \in \omega\right\}$ enumerate the dense subsets of $P$ that are elements of $N$, we produce objects $p_{n}$ and $F_{n}$ that satisfy

(1) $p_{0}=p, F_{0}=\emptyset$,

(2) $p_{n+1} \leq p_{n}$

(3) $p_{n+1} \in N \cap D_{n}$,

(4) $F_{n}$ is a finite subset of $\delta$,

(5) $F_{n+1} \supseteq F_{n}$,

(6) $\left[p_{n+1}\right] \backslash\left[p_{n}\right] \subseteq A_{\delta} \backslash F_{n+1}$,

(7) if $f \in \Phi_{p_{i}}$ for some $i$, then there is an $n$ for which

$$
\left\{\xi \in Y\left(f, p_{n}, p_{i}\right):\left(A_{\delta} \backslash F_{n+1}\right) \cup\{\delta\} \subseteq A_{\xi} \backslash f(\xi)\right\} \text { is stationary. }
$$

At stage $n+1$, we will be handed $F_{n}$ and $p_{n}$, as well as a promise $f \in \Phi_{p_{i}}$ for some $i \leq n$ that we need to take care of.

Since $p_{n}$ and $p_{i}$ are both in $N$, the promise $f^{\prime}:=f\left\lceil Y\left(f, p_{n}, p_{i}\right)\right.$ is in $N$. Clearly $\operatorname{Ban}\left(f^{\prime}\right) \in N$, and since it is nonstationary (by Lemma 6.8) we know $\delta \notin \operatorname{Ban}\left(f^{\prime}\right)$. By definition, this means that

$$
B_{0}:=\left\{\xi \in Y\left(f, p_{n}, p_{i}\right): \delta \in A_{\xi} \backslash f(\xi)\right\} \text { is stationary. }
$$

Since $A_{\delta} \subseteq^{*} A_{\xi} \backslash f(\xi)$ for all $\xi>\delta$ in $\operatorname{dom} f^{\prime}$, there is a finite $F^{\prime}$ for which

$$
B_{1}:=\left\{\xi \in B_{0}: A_{\delta} \backslash F^{\prime} \subseteq A_{\xi} \backslash f(\xi)\right\} \text { is stationary. }
$$

We then set $F_{n+1}=F_{n} \cup F^{\prime}$. Notice that

$$
B_{1} \subseteq\left\{\xi \in Y\left(f, p_{n}, p_{i}\right):\left(A_{\delta} \backslash F_{n+1}\right) \cup\{\delta\} \subseteq A_{\xi} \backslash f(\xi)\right\},
$$

and so we have satisfied condition (7).

Once we have the sequence $\left\{p_{n}: n \in \omega\right\}$, we define

$$
x_{q}=\bigcup_{n \in \omega} x_{p} \cup\{\langle\delta, 1\rangle\},
$$

and for $f \in \Phi_{i}$ (for some $i \in \omega$ ),

$$
K^{+}(f, i)=\left\{\xi \in \operatorname{dom} f:\left[x_{q}\right] \backslash\left[p_{i}\right] \subseteq A_{\xi} \backslash f(\xi)\right\} .
$$


Note that our construction ensures that each $K^{+}(f, i)$ is stationary. Thus if we set

$$
\Phi_{q}=\bigcup_{n \in \omega} \Phi_{p_{n}} \cup \bigcup_{n \in \omega}\left\{f \uparrow K^{+}(f, n): f \in \Phi_{p_{n}}\right\},
$$

then $q:=\left(x_{q}, \Phi_{q}\right)$ is a lower bound for $\left\{p_{n}: n \in \omega\right\}$ in $P$ with $\delta \in[q]$.

To see that $\dot{S}$ is stationary in the generic extension, let us fix a $P$-name $\dot{C}$ for a closed unbounded subset of $\omega_{1}$ and let $p \in P$ be arbitrary. Choose a model $N$ as above with $\dot{C}$ in $N$. An elementary argument shows that any $(N, P)$-generic $q \leq p$ will force that $\delta=N \cap \omega_{1}$ is in $\dot{C}$, and so a condition $q \leq p$ constructed as above will force that $\dot{C} \cap \dot{S} \neq \emptyset$.

We now work toward constructing a model of ZFC in which both the Continuum Hypothesis and $P_{22}$ are true. The notion of forcing just described tells us how to handle a single counterexample to $P_{22}$, and we will appeal to Shelah's theory of $\mathbb{D}$-completeness (Chapter XVIII of [34]) to show that such forcings can be iterated without adding new reals. We turn to this next, dealing first with the requirement of $<\omega_{1}$-properness.

Definition 6.10. A notion of forcing $P$ is said to be $\alpha$-proper if whenever we are given $\mathfrak{N}=\left\langle N_{i}: i \leq \alpha\right\rangle$ and $p$ such that

- $\mathfrak{N}$ is a continuously increasing $\in$-chain of countable elementary submodels of $H(\lambda)$,

- $\left\langle N_{i}: i \leq \beta\right\rangle \in N_{\beta+1}$ for $\beta<\alpha$,

- $P \in N_{0}$,

- $p \in N_{0} \cap P$,

there is a $q \leq p$ that is $\left(N_{i}, P\right)$-generic for all $i \leq \alpha$.

If $P$ is $\alpha$-proper for all $\alpha<\omega_{1}$, then we say that $P$ is $<\omega_{1}$-proper.

Theorem 6.11. The notion of forcing $P$ (as in Theorem 6.9) is $<\omega_{1}-$ proper.

Proof. We prove by induction on $\alpha<\omega_{1}$ that the following statement holds:

$\otimes_{\alpha}$ Given $\mathfrak{N}=\left\langle N_{i}: i \leq \alpha\right\rangle$ and $p \in N_{0} \cap P$ as in Definition 6.10. and $F$ a finite subset of $A_{\delta_{\alpha}}$ (where $\delta_{\alpha}:=N_{\alpha} \cap \omega_{1}$ ), there is a $q \leq p$ that is $\left(N_{i}, P\right)$-generic for all $i \leq \alpha$ that satisfies $[q] \backslash[p] \subseteq A_{\delta_{\alpha}} \backslash F$.

The cases where $\alpha$ is 0 or a successor ordinal are handled by the proof of Theorem 6.9] so we consider the case where $\alpha$ is a limit ordinal. Assume we have $\mathfrak{N}, p$, and $\delta_{\alpha}$ as in $\otimes_{\alpha}$. Let $\left\langle\alpha_{n}: n \in \omega\right\rangle$ be strictly increasing and cofinal in $\alpha$, and define $\delta_{n}=N_{\alpha_{n}} \cap \omega_{1}$. We define a strategy for Player II in the game from Proposition 6.4 (with $N_{\alpha}$ in place of $N$ ) so that together the two players will produce the desired $q$.

The strategy is simple - at stage $n+1$, Player II chooses if possible a $p_{n+1} \leq p_{n}$ satisfying

(1) $p_{n+1} \in N_{\alpha_{n}+1}$,

(2) $\left[p_{n+1}\right] \backslash\left[p_{n}\right] \subseteq A_{\delta_{\alpha}} \cap F_{n+1}$,

(3) $p_{n+1}$ is $\left(N_{i}, P\right)$-generic for all $i \leq \alpha_{n}$.

What we need to show is that Player II's strategy never bogs down. Player II can start by setting $p_{0}=p$, so assume that the strategy has successfully produced $\left\langle p_{i}: i \leq n\right\rangle$ for some $n>0$. Note that $p_{n} \in N_{\alpha_{n-1}+1}$ and $p_{n+1}$ is $\left(N_{i}, P\right)$-generic for all $i \leq \alpha_{n-1}$. 
Player I will respond with his choice of $F_{n+1}$. There is a finite set $F$ such that $A_{\delta_{\alpha_{n+1}}} \backslash F \subseteq A_{\delta_{\alpha}} \backslash F_{n+1}$, and clearly the set $A_{\delta_{\alpha_{n+1}}} \backslash F$ is an element of $N_{\alpha_{n+1}+1}$.

Let $\beta$ be the order-type of $\alpha_{n+1} \backslash \alpha_{n}+1$. Inside the model $N_{\alpha_{n+1}+1}$ we apply our induction hypothesis $\otimes_{\beta}$ to the objects $\left\langle N_{i}: \alpha_{n}+1 \leq i \leq \alpha_{n+1}\right\rangle, p_{n}$, and $A_{\delta_{\alpha_{n+1}}} \backslash F$. This gives us the required $p_{n+1}$, and Player II can continue the game.

Now if Player I uses his winning strategy, the lower bound $q$ will witness that $\otimes_{\alpha}$ holds.

There are two more ingredients needed in order for us to produce a model of $\mathrm{CH}+P_{22}$. Both of these happen to be very technical conditions that require several pages to define properly, and since the proofs that our notion of forcing satisfies these conditions are routine modifications of the corresponding arguments from [3], at the urging of the referee we will be content with simply stating the results.

Theorem 6.12. Let $P$ be the notion of forcing from Theorem 6.9,

(1) $P$ is $\mathbb{D}$-complete for a simple $\aleph_{0}\left(\right.$ even $\aleph_{1}$ ) completeness system (page 227 of 34 ).

(2) P satisfies the $\aleph_{2}-$ p.i.c. (page 409 of 34]).

Proof. See Lemma 3.5 and Lemma 3.6 of $[3]$.

We now return to the construction of a model of ZFC in which both $\mathrm{CH}$ and $P_{22}$ are true. We have indicated that the model is the result of forcing with a countable support iteration $\mathbb{P}=\left\langle P_{\alpha}, \dot{Q}_{\alpha}: \alpha<\omega_{1}\right\rangle$, where at each stage we kill off a potential counterexample to $P_{22}$ using the notion of forcing outlined earlier.

Our work together with Theorem V.7.1 of [34] implies that the limit of our iteration, $P_{\omega_{2}}$, is a totally proper notion of forcing with the $\aleph_{2}$-chain condition, so that the Continuum Hypothesis remains true in $V\left[G_{\omega_{2}}\right]$. This implies that any counterexample to $P_{22}$ in $V\left[G_{\omega_{2}}\right]$ is actually $\aleph_{1}$-generated. Standard bookkeeping arguments allow us to make sure that any such potential counterexample is killed off at some earlier stage of our iteration. Note also that once a counterexample has been killed, it is not resurrected at later stages of our iteration because totally proper forcing preserves stationary sets and adds no new countable sets to the ground model. Thus we may conclude that in $V\left[G_{\omega_{2}}\right]$ both $\mathrm{CH}$ and $P_{22}$ holds.

It is straightforward to modify the partial order $P$ to obtain stronger results. For example, we can prove the consistency with $\mathrm{CH}$ of the statement that for every $P$-ideal $\mathcal{I}$ in $\left[\omega_{1}\right]^{\aleph_{0}}$ and every stationary $S \subseteq \omega_{1}, S$ has a stationary subset $S^{\prime}$ such that either $\left[S^{\prime}\right]^{\aleph_{0}} \subseteq \mathcal{I}$ or $\left[S^{\prime}\right]^{\aleph_{0}} \cap \mathcal{I}=\emptyset$.

\section{REFERENCES}

[1] A. V. Arhangel'skiĭ and R. Z. Buzyakova, Convergence in compacta and linear Lindelöfness, Comment. Math. Univ. Carolinae 39, 1 (1998) 159-166. MR.1623006 (99c:54034)

[2] U. Abraham and S. Todorčević, Consistency with CH of a combinatorial principle that implies there are no Suslin trees and all $\left(\omega_{1}, \omega_{1}\right)$-gaps are Hausdorff, preprint.

[3] , Partition properties of $\omega_{1}$ compatible with CH, Fund. Math. 152 (1997) 165-181. MR:1441232 (98b:03064)

[4] K. Devlin and S. Shelah, Suslin properties and tree topologies, Proc. London Math. Soc. 39 (1979) 237-252. MR548979 (80m:54031)

[5] E. K. van Douwen, The integers and topology, in: Handbook of Set-Theoretic Topology, K. Kunen and J. Vaughan ed., North-Holland (1984) 111-167. MR776619 (85k:54001)

[6] T. Eisworth, CH and first countable, countably compact spaces, Topology Appl. 109 (2001), no. 1, 55-73. MR.1804563(2001k:54010) 
[7] _ Totally proper forcing and the Moore-Mrówka problem, Fund. Math. 177 (2003), no. 2, 121-136. MR.1992528 (2004g:03093)

[8] T. Eisworth and P. Nyikos, First countable, countably compact spaces and the continuum hypothesis, Trans. Amer. Math. Soc. 357 (2005), 4269-4299. MR2156711 (2006g:03091)

[9] T. Eisworth and J. Roitman, CH with no Ostaszewski spaces, Trans. Amer. Math. Soc. 351 (1999), no. 7, 2675-2693. MR1638230 (2000b:03182)

[10] R. Engelking, General Topology, Heldermann-Verlag, Berlin, 1989. MR.1039321 (91c:54001)

[11] G. Gruenhage, Some results on spaces having an orthobase or a base of subinfinite rank, Proceedings of the 1977 Topology Conference (Louisiana State Univ., Baton Rouge, La., 1977) I. Topology Proc. 2 (1977), no. 1, 151-159. MR540602 (80k:54056)

[12] G. Gruenhage, Paracompactness and subparacompactness in perfectly normal locally compact spaces, Usp. Mat. Nauk 35, no. 3 (1980) 44-49 (Russian) International Topology Conference (Moscow State Univ., Moscow, 1979). Translated from the English by A. V. Arhangel'skiü. MR:580619 (81k:54021)

[13] N. Howes, A note on transfinite sequences, Fund. Math. 106 (1980) 213-216. MR.584493 (82c:54002)

[14] J. Hirschorn, Random trees under CH, Israel J. Math. 157 (2007) 123-154. MR2342443 (2008g:03084)

[15] Tetsuya Ishiu, $\alpha$-properness and Axiom A, Fund. Math. 186 (2005), no. 1, 25-37. MR2163100 (2006g:03081)

[16] N. N. Jakovlev, On the theory of o-metrizable spaces, Dokl. Akad. Nauk. SSSR 229 (1976) 1330-1331. MR0415578 (54:3663)

[17] I. Juhász, Consistency results in topology, in: Handbook of Mathematical Logic, J. Barwise ed., North-Holland, 1977, 503-522. MR0457132 (56:15351)

[18] I. Juhász, L. Soukup, and S. Szentmiklóssy, What is left of $\mathrm{CH}$ after you add Cohen reals? Top. Appl. 85 (1998) 165-174. MR1617461 (99d:54001)

[19] K. Kunen, Locally compact linearly Lindelöf spaces, Comment. Math. Univ. Carolinae 43 (2002) 155-158. MR1903314 (2003d:54040)

[20] P. J. Nyikos, Subsets of ${ }^{\omega} \omega$ and the Fréchet-Urysohn property, Top. Appl. 48 (1992) 91-116. MR1195504 (93k:54011)

[21] _ Various topologies on trees, in: Proceedings of the Tennessee Topology Conference, P. R. Misra and M. Rajagopalan, eds., World Scientific Publishing Co., 1997, 167-198. MR1607401 (98m:54037)

[22] - The theory of nonmetrizable manifolds, in: Handbook of Set-Theoretic Topology, K. Kunen and J. Vaughan ed., North-Holland, 1984, 633-684. MR776619 (85k:54001)

[23] _ Applications of some strong set-theoretic axioms to locally compact $T_{5}$ and hereditarily scwH spaces, Fund. Math. 176 (1) (2003) 25-45. MR1971471(2004k:54008)

[24] _ Hereditarily normal, locally compact Dowker spaces, Topology Proceedings 24 (1999) 261-276. MR.1802691 (2001i:54017)

[25] _ Correction to "Complete normality and metrization theory of manifolds," Top. Appl. 138 (2004) 325-327. MR2035491 (2004k:54030)

[26] - Applications of antidiamond and anti-PFA axioms to metrization of manifolds, preprint.

[27] T. Przymusinski, Products of normal spaces, in: Handbook of Set-Theoretic Topology, K. Kunen and J. Vaughan ed., North-Holland, 1984, 781-826. MR776637 (86c:54007)

[28] E. Pearl, Linearly Lindelöf problems, in: Open Problems in Topology II, E. Pearl, ed., Elsevier B.V., 2007, 225-231. MR2367385

[29] J. Roitman, Basic $S$ and L, in: Handbook of Set-Theoretic Topology, K. Kunen and J. Vaughan, ed., North-Holland, 1984, pp. 295-326. MR776626 (87a:54043)

[30] M. E. Rudin, Lectures on Set Theoretic Topology, CBMS Regional Conference Series No. 23, American Mathematical Society, Providence, RI, 1977. MR0367886 (51:4128)

[31] _ The undecidability of the existence of a perfectly normal nonmetrizable manifold, Houston J. Math. 5 (1979) 105-112. MR.546759 (80j:54014)

[32] — A nonmetrizable manifold from $\diamond^{+}$, Top. Appl. 28 (1988) 105-112. MR932975 (89c:54049)

[33] M. E. Rudin and P. L. Zenor, A perfectly normal nonmetrizable manifold, Houston Math. J. 2 (1976) 129-134. MR0394560 (52:15361) 
[34] S. Shelah, Proper and improper forcing, Perspectives in Mathematical Logic, Springer, 1998. MR.1623206 (98m:03002)

[35] C. Schlindwein, Suslin's hypothesis does not imply stationary antichains, Ann. Pure and Appl. Logic 32 (1993) 153-167. MR.1241252 (94i:03101)

[36] $\_$SH plus $\mathrm{CH}$ does not imply stationary antichains, Ann. Pure and Appl. Logic 124 (2003) 233-265. MR2013399 (2004j:03056)

[37] S. Todorčević, A dichotomy for P-ideals of countable sets, Fund. Math. 166 (2000) 251-267. MR:1809418 (2001k:03111)

Department of Mathematics, University of Northern Iowa. Cedar Falls, Iowa 50614

E-mail address: eisworth@math.uni.edu

Department of Mathematics, University of South Carolina, Columbia, South CarOLINA

E-mail address: nyikos@math.sc.edu 